\title{
BMJ Open Lipoprotein(a) plasma levels, bone mineral density and risk of hip fracture: a post hoc analysis of the Women's Health Initiative, USA
}

\author{
Bernhard Haring, ${ }^{1}$ Carolyn J Crandall, ${ }^{2}$ Laura Carbone, ${ }^{3}$ Simin Liu, ${ }^{4}$ Wenjun Li, ${ }^{5}$ \\ Karen C Johnson, ${ }^{6}$ Jean Wactawski-Wende, ${ }^{7}$ Aladdin $\mathrm{H}$ Shadyab, ${ }^{8}$ \\ Margery L Gass, ${ }^{9}$ Victor Kamensky, ${ }^{10}$ Jane A Cauley, ${ }^{11}$ Sylvia Wassertheil-Smoller ${ }^{10}$
}

To cite: Haring B, Crandall CJ, Carbone L, et al. Lipoprotein(a) plasma levels, bone mineral density and risk of hip fracture: a post hoc analysis of the Women's Health Initiative, USA. BMJ Open 2019;9:e027257. doi:10.1136/ bmjopen-2018-027257

- Prepublication history for this paper is available online. To view these files, please visit the journal online (http://dx.doi org/10.1136/bmjopen-2018027257).

Received 13 October 2018 Revised 19 February 2019 Accepted 21 February 2019
Check for updates

(C) Author(s) (or their employer(s)) 2019. Re-use permitted under CC BY-NC. No commercial re-use. See rights and permissions. Published by BMJ.

For numbered affiliations see end of article.

Correspondence to Dr Bernhard Haring; Haring_B@ukw.de

\section{ABSTRACT}

Objectives Elevated Lipoprotein(a) (Lp[a]) is a well-known risk factor for cardiovascular disease. However, its roles in bone metabolism and fracture risk are unclear. We therefore investigated whether plasma $L p(a)$ levels were associated with bone mineral density (BMD) and incident hip fractures in a large cohort of postmenopausal women. Design Post hoc analysis of data from the Women's Health Initiative (WHI), USA.

Setting 40 clinical centres in the USA.

Participants The current analytical cohort consisted of 9698 white, postmenopausal women enrolled in the WHI, a national prospective study investigating determinants of chronic diseases including heart disease, breast and colorectal cancers and osteoporotic fractures among postmenopausal women. Recruitment for WHI took place from 1 October 1993 to 31 December 1998.

Exposures Plasma $\mathrm{Lp}(\mathrm{a})$ levels were measured at baseline.

Outcome measures Incident hip fractures were ascertained annually and confirmed by medical records with follow-up through 29 August 2014. BMD at the femoral neck was measured by dual X-ray absorptiometry in a subset of participants at baseline.

Statistical analyses Cox proportional hazards and logistic regression models were used to evaluate associations of quartiles of plasma $L p(a)$ levels with hip fracture events and hip BMD T-score, respectively. Results During a mean follow-up of 13.8 years, 454 incident cases of hip fracture were observed. In analyses adjusting for confounding variables including age, body mass index, history of hysterectomy, smoking, physical activity, diabetes mellitus, general health status, cardiovascular disease, use of menopausal hormone therapy, use of bisphosphonates, calcitonin or selectiveoestrogen receptor modulators, baseline dietary and supplemental calcium and vitamin D intake and history of fracture, no significant association of plasma Lp(a) levels with low hip BMD T-score or hip fracture risk was detected.

Conclusions These findings suggest that plasma $L p(a)$ levels are not related to hip BMD T-score or hip fracture events in postmenopausal women.

Trial registration number NCT00000611; Post-results.
Strengths and limitations of this study

- Large, geographically diverse prospective cohort with long duration of follow-up.

- Detailed assessment of risk factors for fracture events and physician-adjudicated information on hip fractures was available.

- The analysis was restricted to white postmenopausal women and thus generalisability is limited.

- No information on apolipoprotein(a) size was available.

- Residual confounding cannot be excluded.

\section{INTRODUCTION}

Lipoprotein(a) $[\mathrm{Lp}(\mathrm{a})]$ is a plasma lipoprotein consisting of a cholesterol-rich low-density lipoprotein (LDL)-like particle having one molecule of apolipoprotein B100 and an additional protein, apolipoprotein(a), attached to apoB via a disulfide bond. Variability of $\operatorname{Lp}(\mathrm{a})$ is mainly genetically determined with similar levels in men and women but differences among races. ${ }^{1}$ Elevated $\mathrm{Lp}$ (a) plasma levels have been identified to be an independent risk factor for cardiovascular events and aortic valve stenosis. ${ }^{2}$ The mechanisms of how elevated $\operatorname{Lp}(\mathrm{a})$ lead to adverse cardiac outcomes are not completely understood, but several mechanisms and theories have been proposed. ${ }^{34}$ Among the most accepted, $\operatorname{Lp}(\mathrm{a})$ because of its structural/functional homology to plasminogen competes with plasminogen and impairs fibrinolysis and pericellular proteolysis. Moreover, elevated Lp(a) concentration may not only decrease fibrinolytic activity, it may also prolong plasminogen activation by preventing the irreversible inhibition of the plasminogen activators. ${ }^{5}$

There is accumulating evidence that $\operatorname{Lp}(\mathrm{a})$ and plasminogen, besides their roles in 
haemostasis and cardiovascular disease, may also be essential for tissue repair. Plasminogen deficiency has been recently shown to inhibit fracture repair and adequate fibrinolysis is increasingly regarded as an emerging target to fracture healing. ${ }^{6}$ It is therefore plausible that $\mathrm{Lp}(\mathrm{a})$ plasma levels may also contribute to healing processes and remodelling from traumas or injuries. Furthermore, elevated Lp(a) plasma levels have been associated with aortic valve stenosis as well as chronic kidney disease, both of which increase the likelihood of syncopal episodes, falls and decreased bone mineral density (BMD) ${ }^{78}$

To this date, evidence on the associations between $\mathrm{Lp}$ (a) and BMD is sparse, and prior findings have been conflicting. ${ }^{910}$ To our knowledge, no previous study has investigated the association between Lp(a), BMD and hip fracture risk. We thus aimed to investigate if $L p(a)$ levels are associated with hip BMD T-scores and hip fracture events in a large cohort of white postmenopausal women with long-term follow-up.

\section{METHODS}

\section{Study population}

The study population consisted of women enrolled in the Women's Health Initiative (WHI). WHI is a long-term health study that has focused on strategies for preventing heart disease, breast and colorectal cancers, and osteoporotic fractures in postmenopausal women. ${ }^{11}$ It has two major parts: a clinical trial (CT) and an observational study (OS). Recruitment to WHI was conducted by 40 participating clinical sites nationwide in the USA and took place from 1 October 1993 to 31 December 1998, using such methods as media advertising, direct population mailing, community lectures and public service announcements, and celebrity endorsements. Women were eligible to participate if 50-79 years of age and postmenopausal at the time of enrolment. In addition to age and menopausal status, women were generally free of recent serious cardiac, pulmonary, renal and hepatic conditions and had at least 3 years of life expectancy. The WHI OS examined the predictors and natural course of important causes of morbidity and mortality in postmenopausal women. The three WHI CTs tested several interventions among postmenopausal women: a low-fat eating pattern, menopausal hormone therapy and calcium and vitamin D supplementation. Institutional review boards (IRBs) of all participating institutions (40 clinical site IRBs, the coordinating centre IRB and ethical review at National Institutes of Health) approved all protocols and all participants provided written informed consent.

\section{Lp(a) levels}

A subsample of women enrolled in WHI, randomly selected at baseline, provided fasting blood specimens at baseline which were collected following a standardised protocol. ${ }^{11}$ Blood samples were labelled, centrifuged and frozen on site in $-70^{\circ} \mathrm{C}$ freezers and later shipped to the central WHI specimen repository. Lp(a) was quantitated using an isoform independent bi-site ELISA assay procedure based on the linkage of apo(a) to apoB. ${ }^{12}$ Standardisation and ongoing quality control was established and maintained with Northwest Lipid Research Clinic. Throughout the study, the laboratory participated in and remained certified by the National Heart, Lung, and Blood Institute, Centers for Disease Control Part III programme. ${ }^{13}$ As $\mathrm{Lp}(\mathrm{a})$ levels vary by race and due to a limited number of non-white participants to reliably examine associations, we limited this analysis to white participants who were followed up through 29 August $2014(\mathrm{n}=9698)$.

\section{Outcomes}

The primary outcome measure was incident hip fracture events. Information regarding incident fractures was determined by self-report semiannually through the end of the WHI CTs and annually when the trials finished. In the WHI OS, fractures were self-reported annually. All self-reported hip fractures were subsequently confirmed by medical record review by blinded physician adjudicators. ${ }^{11}$

\section{Covariate assessment}

Information on age, personal history of fracture, smoking status, physical activity and general health status were obtained by self-report questionnaires at baseline. ${ }^{11}$ Trained clinic staff measured weight and height at baseline using standardised protocols. Body mass index (BMI) was calculated as weight in kilograms divided by the square of the height in metres. Current medication or supplement use was assessed at baseline by clinic interviewers. Diabetes status was determined by self-reported physician diagnosis of diabetes treated with either oral medication or insulin. Cardiovascular Disease (CVD) was defined as a reported history of cardiovascular disease, myocardial infarction, stroke, transitory ischaemic attack, angina or revascularisation. BMD at the femoral neck was measured by dual X-ray absorptiometry (QDR 2000, $2000+$ or 4500 W, Hologic, Massachusetts, USA) in a subset of WHI participants (WHI-BMD cohort) at 3 of the 40 US clinical centres (Pittsburgh, Pennsylvania; Birmingham, Alabama; and Tucson/Phoenix, Arizona, USA) at baseline $(\mathrm{n}=758) .{ }^{11}$ For this analysis, BMD T-scores were based on the Third National Health and Nutrition Examination Survey (NHANES III) reference database.

\section{Statistics}

Baseline characteristics across quartiles of $\operatorname{Lp}(\mathrm{a})$ plasma levels were compared using $\chi^{2}$ statistics for categorical variables and ANOVA, as appropriate, for continuous variables. HRs for risk of hip fracture associated with quartiles of Lp(a) were obtained using covariate-adjusted Cox proportional hazards models. Among women who experienced a fracture event, duration of follow-up was defined as time to first fracture. Among women who did not experience a fracture during follow-up, duration of follow-up was defined as time until last follow-up visit, or death, whichever came 


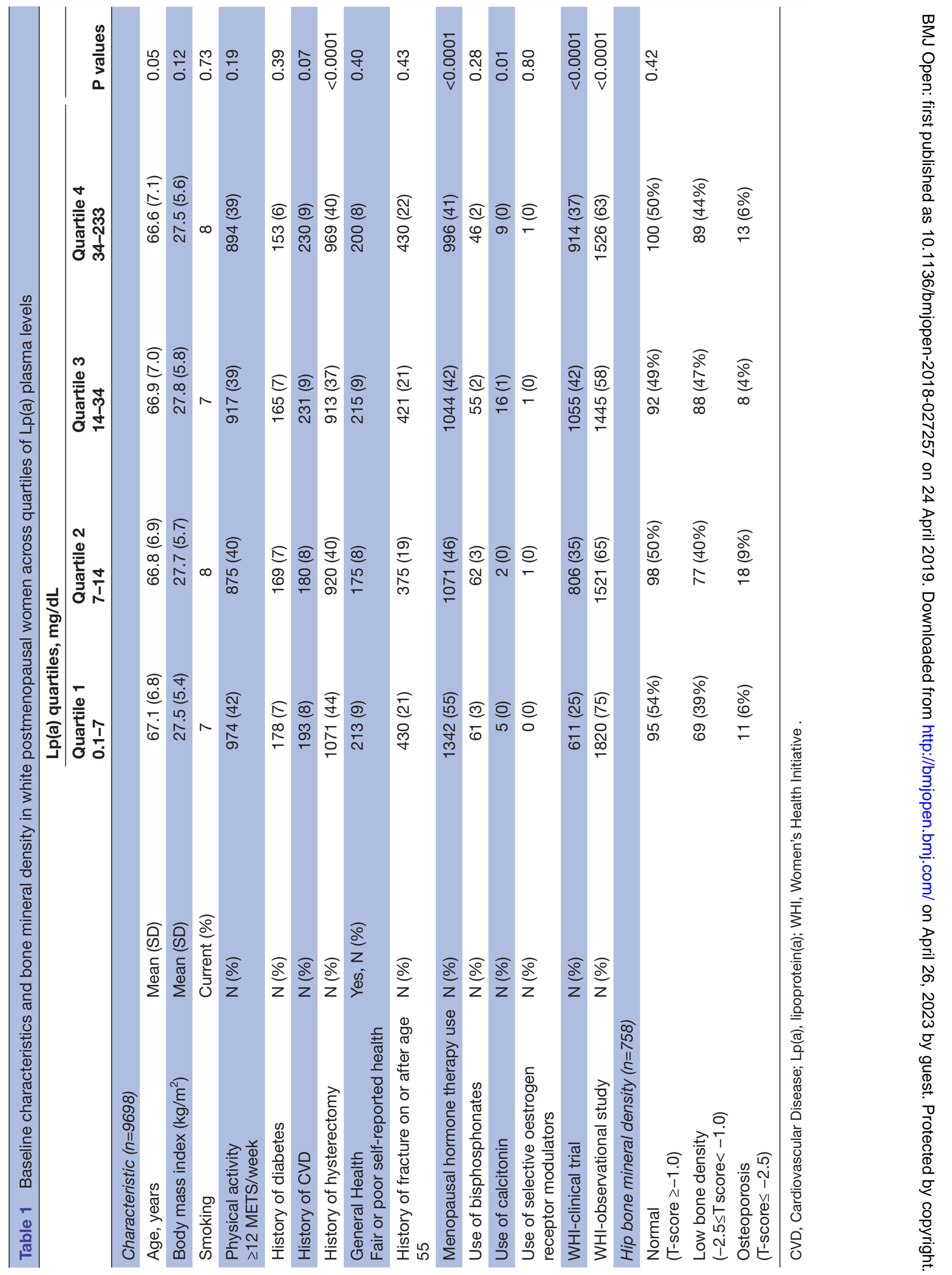


Table 2 HRs for incident hip fracture events by quartiles of $L p(a)$ plasma levels

\begin{tabular}{|c|c|c|c|c|c|c|}
\hline Quartiles (Q) & $\begin{array}{l}\text { Median Lp(a) } \\
\mathrm{mg} / \mathrm{dL}\end{array}$ & $\begin{array}{l}\text { No of } \\
\text { individuals }\end{array}$ & No of cases & HR $(95 \% \mathrm{Cl})^{\star}$ & HR $(95 \%$ Cl)† & HR (95\% Cl)‡ \\
\hline Q1 & 3 & 2431 & 113 & 1 & 1 & 1 \\
\hline Q3 & 20 & 2500 & 122 & $1.06(0.82$ to 1.37$)$ & $0.99(0.76$ to 1.29$)$ & $0.98(0.75$ to 1.28$)$ \\
\hline Q4 & 59 & 2440 & 114 & 1.05 (0.81 to 1.36$)$ & 0.99 (0.76 to 1.30$)$ & $0.99(0.76$ to 1.30$)$ \\
\hline $\mathrm{P}$ trend & & & & 0.60 & 0.93 & 0.94 \\
\hline
\end{tabular}

${ }^{*}$ Model 1 adjusted for linear age and BMI.

†Model 2 additionally adjusted for WHI CT assignment, smoking, physical activity, diabetes mellitus, general health status, history of fracture, baseline dietary and supplemental vitamin D intake, baseline dietary and supplemental calcium intake, use of menopausal hormone therapy and use of bisphosphonates, calcitonin or selective-oestrogen receptor modulators.

$\ddagger$ Model 3 adjusted for Model two and additionally for history of hysterectomy and CVD.

BMI, body mass index; CVD, Cardiovascular Disease; Lp(a), lipoprotein(a); WHI, Women's Health Initiative.

first. Three models were formed to examine the effect of potential confounding: Model 1 included age and BMI as covariates. Model 2 additionally adjusted for WHI CT assignment, smoking, physical activity, diabetes mellitus, history of fracture on or after age 55 , general health status, baseline dietary and supplemental vitamin D intake, baseline dietary and supplemental calcium intake, use of menopausal hormone therapy (by self-report OS or by CT randomisation), use of bisphosphonates, calcitonin, or selective-oestrogen receptor modulators. Model 3 adjusted for all variables in Model 2 and additionally for history of CVD and history of hysterectomy. Continuous predictors were handled as if linear except for vitamin $\mathrm{D}$ and calcium intake, which were categorised $(<200,200-<400,400-6600$ and $\geq 600 \mathrm{IU} /$ day for vitamin $\mathrm{D}$ and $<800,800<1200$ or $\geq 1200 \mathrm{mg} /$ day for calcium). Using the aforementioned modelling approach, we used logistic regression models to examine the associations of $\mathrm{Lp}$ (a) plasma levels with hip BMD T-score, categorised as either normal BMD (T-score $\geq-1$ ) and low BMD (T-score $<-1)$ in the analysis. The reference category for the outcome in this analysis was normal BMD (T-score $\geq-1$ ). Finally, we examined the association of quartiles of $\operatorname{Lp}(\mathrm{a})$ plasma levels with risk of any non-hip fracture event using Cox proportional hazards regression models. Statistical analyses were conducted using SAS V.9.4.

\section{Sample size calculation}

To our knowledge, data on the association between $\operatorname{Lp}(\mathrm{a})$ plasma levels and risk of hip fractures are missing. Our study population consisted of 9698 women with $\mathrm{Lp}(\mathrm{a})$ measurements; 454 incident cases of hip fractures were documented. The sample size was sufficient to detect a HR of greater than 1.3 with $80 \%$ power at a two-sided significance level of $\alpha=0.05$ for a two group comparison.

\section{Patient and public involvement}

WHI was designed to address the gaps in knowledge about the major health issues in postmenopausal women. Patients assisted research staff in recruiting, and results for all measures done at the study examinations were explained to each participant. Major study results were communicated to participants via newsletters.

\section{RESULTS}

The overall analytic sample consisted of 9698 women. Across quartiles of $\operatorname{Lp}(\mathrm{a})$, women were approximately the

Table 3 ORs for low bone mineral density (T-score <-1.0) by quartiles of Lp(a) plasma levels

\begin{tabular}{|c|c|c|c|c|c|c|}
\hline Quartiles (Q) & $\begin{array}{l}\text { Median Lp(a) } \\
\mathrm{mg} / \mathrm{dL}\end{array}$ & $\begin{array}{l}\text { No. of } \\
\text { individuals }\end{array}$ & No. of cases & OR $(95 \% \mathrm{Cl})^{*}$ & OR $(95 \% \mathrm{Cl}) \dagger$ & OR (95\% Cl)‡ \\
\hline Q1 & 3 & 175 & 80 & 1 & 1 & 1 \\
\hline Q3 & 20 & 188 & 96 & 1.52 (0.96 to 2.42 ) & 1.60 (0.96 to 2.66$)$ & 1.60 (0.96 to 2.66$)$ \\
\hline Q4 & 59 & 202 & 102 & 1.30 (0.83 to 2.06$)$ & $1.36(0.81$ to 2.26$)$ & 1.35 (0.81 to 2.25$)$ \\
\hline $\mathrm{P}$ trend & & & & 0.16 & 0.17 & 0.17 \\
\hline
\end{tabular}

The reference category for the outcome in this analysis was normal BMD (T-score $\geq-1$ ).

${ }^{*}$ Model 1adjusted for linear age and BMI.

†Model 2additionally adjusted for WHI CT assignment, smoking, physical activity, diabetes mellitus, general health status, history of fracture, baseline dietary and supplemental vitamin $\mathrm{D}$ intake, baseline dietary and supplemental calcium intake, use of menopausal hormone therapy and use of bisphosphonates, calcitonin or selective-oestrogen receptor modulators.

$\ddagger$ Model 3 adjusted for Model two and additionally for history of hysterectomy and CVD.

BMD, bone mineral density; BMI, body mass index; CVD, Cardiovascular Disease; Lp(a), lipoprotein(a); WHI, Women's Health Initiative. 
Table 4 HRs for incident non-hip fracture events by quartiles of Lp(a) plasma levels

\begin{tabular}{|c|c|c|c|c|c|c|}
\hline Quartiles (Q) & $\begin{array}{l}\text { Median Lp(a) } \\
\text { mg/dL }\end{array}$ & $\begin{array}{l}\text { No of } \\
\text { individuals* }\end{array}$ & No of cases & HR $(95 \% \mathrm{Cl}) \dagger$ & HR (95\% Cl)‡ & HR $(95 \% \mathrm{Cl}) \S$ \\
\hline Q1 & 3 & 2418 & 711 & 1 & 1 & 1 \\
\hline Q2 & 10 & 2319 & 636 & $0.92(0.82$ to 1.02$)$ & 0.88 (0.79 to 0.99$)$ & $0.88(0.79$ to 0.98$)$ \\
\hline Q3 & 20 & 2493 & 773 & 1.05 (0.94 to 1.16$)$ & $1.00(0.90$ to 1.11$)$ & $0.99(0.89$ to 1.11$)$ \\
\hline Q4 & 59 & 2428 & 687 & $0.96(0.86$ to 1.06$)$ & 0.91 (0.81 to 1.01$)$ & $0.90(0.81$ to 1.01$)$ \\
\hline $\mathrm{P}$ trend & & & & 0.99 & 0.33 & 0.98 \\
\hline
\end{tabular}

*Missing information on 40 individuals.

†Model 1 adjusted for linear age and BMI.

¥Model 2 additionally adjusted for WHI CT assignment, smoking, physical activity, diabetes mellitus, general health status, history of fracture, baseline dietary and supplemental vitamin D intake, baseline dietary and supplemental calcium intake, use of menopausal hormone therapy and use of bisphosphonates, calcitonin or selective-oestrogen receptor modulators.

$\S$ Model 3 adjusted for Model two and additionally for history of hysterectomy and CVD.

BMI, body mass index; CVD, Cardiovascular Disease; WHI, Women's Health Initiative.

same age (67 years) and slightly overweight (mean BMI $27 \mathrm{~kg} / \mathrm{m}^{2}$ ) (table 1). Furthermore, there were no marked differences in history of diabetes, general health, history of CVD, history of fracture on or after age 55, smoking or physical activity. Women with $\operatorname{Lp}(\mathrm{a})$ in the lower quartiles were more likely to use menopausal hormone therapy and to have had a history of hysterectomy compared with women with high plasma $\operatorname{Lp}(\mathrm{a})$. The use of bisphosphonates, calcitonin and selective oestrogen receptor modulators was low. No clinically significant differences in hip BMD were found across $\mathrm{Lp}(\mathrm{a})$ quartiles.

Included women (WHI-OS and WHI-CT) were followed for a mean (SD) of 13.8 years (5.8); women in the WHI-OS were followed for 13.3 years (5.7); women in WHI-CT were followed for 14.7 years $(5.8) \quad(\mathrm{p}<0.0001)$. There were 454 cases of hip fractures in the overall analytic sample. No significant associations between quartiles of plasma $\mathrm{Lp}(\mathrm{a})$ levels and hip fractures were detected (table 2). Further, there were no significant associations between $\operatorname{Lp}(\mathrm{a})$ plasma levels and hip BMD T-score (table 3). Finally, no association between quartiles of plasma $\operatorname{Lp}(\mathrm{a})$ levels and any non-hip fracture event was seen (table 4).

\section{DISCUSSION}

Our results based on a large population of white postmenopausal women suggest that plasma $\operatorname{Lp}(\mathrm{a})$ levels are not associated with BMD T-score or hip fracture risk. These findings complement prior epidemiological studies on the associations of lipid and lipoproteins with BMD and fracture risk. ${ }^{14-20}$

Higher triglyceride or LDL cholesterol levels in midlife and in postmenopausal women have been associated with increased fracture risk. ${ }^{145}$ Moreover, some authors reported a modest, inverse relationship between lumbar spine and whole body BMD with serum triglyceride and LDL levels in postmenopausal women and HDL in premenopausal women but overall data on the relationship between lipid profiles and BMD are conflicting. ${ }^{16-20}$ Meta-analyses of randomised controlled trials found lipid-lowering therapies to be associated with significant increases in BMD but not associated with fracture risk. ${ }^{21}$ Little is known on the associations between Lp(a) and BMD. ${ }^{9}{ }^{10}$ Elevated Lp(a) plasma levels have been associated with lower BMD in early postmenopausal overweight women in some populations but questions remain. ${ }^{9}$ These questions involve inter alia the concurrent use of hormone therapy as well as concomitant chronic disease: Oestrogen but not addition of progestogen has been shown to lower plasma $\mathrm{Lp}(\mathrm{a})$ levels most likely due to a reduction in the rate of apolipoprotein(a) synthesis. ${ }^{22}$ This is clinically relevant as the relationship of elevated $\mathrm{Lp}$ (a) levels with cardiovascular disease risk has been further shown to be modified by oestrogen therapy and that the predictive utility of $\operatorname{Lp}(\mathrm{a})$ was markedly attenuated among women taking oestrogen. ${ }^{23}$ On the other hand, an increase in $\mathrm{Lp}$ (a) concentrations is seen in non-nephrotic patients with primary renal disease even in the earliest stage when GFR is not yet subnormal. ${ }^{8}$ Elevated $\mathrm{Lp}$ (a) levels have been shown to be associated with aortic valve stenosis in a large Danish cohort. ${ }^{7}$ As aortic valve stenosis increases the likelihood of syncopal episodes, an increased fracture risk may be the consequence. Our results show a higher prevalence of hormone therapy use among women in the lower quartiles of $\operatorname{Lp}(\mathrm{a})$ plasma levels but no clinically meaningful differences in hip T-scores across $\mathrm{Lp}$ (a) quartiles. We also did not see any association of $\mathrm{Lp}$ (a) with low BMD T-score. Moreover, after adjusting for a wide range of confounding factors, we did not find any association of $\mathrm{Lp}(\mathrm{a})$ plasma levels with either hip fracture or non-hip fracture events.

Strengths of this study include a long duration of follow-up, detailed information regarding risk factors for fracture events and adjudicated information regarding hip fractures. Nonetheless, there are several limitations. First, our analysis was restricted to white postmenopausal women, thus, generalisability is limited. Second, we are lacking information on kidney function and on apolipoprotein(a) size. Smaller apolipoprotein (a) isoforms and higher Lp(a) levels have been linked to incident cardiovascular disease, but their independent contributions are less clear. ${ }^{24} 25$ Third, due to the observational character of this study, although 
our analyses included women who were generally healthy at baseline, residual confounding cannot be excluded. Finally, although our sample size was large, our study may have lacked power to detect small differences.

\section{Author affiliations}

${ }^{1}$ Department of Internal Medicine I, University of Würzburg, Würzburg, Germany ${ }^{2}$ Department of Medicine, University of California, Los Angeles, California, USA ${ }^{3}$ Department of Medicine, Augusta University Medical College of Georgia, Augusta, Georgia, USA

${ }^{4}$ Department of Epidemiology and Medicine, Brown University, Providence, Rhode Island, USA

${ }^{5}$ Department of Medicine, University of Massachusetts Medical School, Worcester, Massachusetts, USA

${ }^{6}$ Department of Preventive Medicine, University of Tennessee Health Science Center, Memphis, Tennessee, USA

${ }^{7}$ Department of Epidemiology and Environmental Health, University at Buffalo, Buffalo, New York, USA

${ }^{8}$ Department of Family Medicine and Public Health, University of California San Diego, La Jolla, California, USA

${ }^{9}$ North American Menopause Society, Cleveland, Ohio, USA

${ }^{10}$ Department of Epidemiology and Population Health, Albert Einstein College of Medicine, Bronx, New York, USA

${ }^{11}$ Department of Epidemiology, University of Pittsburgh, Pittsburgh, Pennsylvania, USA

Acknowledgements The authors thank the WHI investigators, staff and the study participants for their outstanding dedication and commitment. Program Office (National Heart, Lung, and Blood Institute, Bethesda, Maryland): Jacques Rossouw, Shari Ludlam, Joan McGowan, Leslie Ford and Nancy Geller Clinical Coordinating Center (Fred Hutchinson Cancer Research Center, Seattle, Washington): Garnet Anderson, Ross Prentice, Andrea LaCroix, and Charles Kooperberg Investigators and Academic Centers (Brigham and Women's Hospital, Harvard Medical School, Boston, Massachusetts): JoAnn E. Manson (MedStar Health Research Institute/ Howard University, Washington, DC); Barbara V. Howard (Stanford Prevention Research Center, Stanford, California); Marcia L. Stefanick (The Ohio State University, Columbus, Ohio); Rebecca Jackson (University of Arizona, Tucson/ Phoenix, Arizona); Cynthia A. Thomson (University at Buffalo, Buffalo, New York); Jean Wactawski-Wende (University of Florida, Gainesville/Jacksonville, Florida); Marian Limacher (University of lowa, lowa City/Davenport, lowa); Jennifer Robinson (University of Pittsburgh, Pittsburgh, Pennsylvania); Lewis Kuller (Wake Forest University School of Medicine, Winston-Salem, North Carolina); Sally Shumaker (University of Nevada, Reno, Nevada); Robert Brunner (University of Minnesota, Minneapolis, Minnesota); Karen L. Margolis Women's Health Initiative Memory Study (Wake Forest University School of Medicine, Winston-Salem, North Carolina): Mark Espeland.

Contributors Study design and study conduct: BH and SW-S. Data collection: SL, JW-W, JAC and SW-S. Data analysis: BH, SW-S and VK. Data interpretation: BH, CJC, LC, SL, WL, KCJ, JW-W, AHS, MLG, VK and JAC. Drafting manuscript: BH, JAC, CJC, SL, LC, AHS, MLG, JW-W, WL, KCJ and SW-S. Revising manuscript content: BH, JAC, CJC, SL, LC, AHS, MLG, JW-W, WL, KCJ, VK and SW-S. Approving final version of manuscript: all authors. BH, VK and SW-S take responsibility for the integrity of the data analysis.

Funding The WHI program is funded by the National Heart, Lung, and Blood Institute, National Institutes of Health, U.S. Department of Health and Human Services through contracts HHSN268201600018C, HHSN268201600001C, HHSN268201600002C, HHSN268201600003C and HHSN268201600004C. The opinions expressed in this manuscript are those of the authors and do not necessarily reflect the views of the Department of Health and Human Services/ National Institutes of Health. The Women's Health Initiative (WHI) project office at the National Heart, Lung, and Blood Institute (NHLBI), which was the sponsor, had a role in the design and conduct of the study and in the collection and management of the data. The sponsor did not have a role in analysis and interpretation of the data, the preparation of the manuscript or the decision to submit the manuscript for publication. Review and approval of the manuscript was carried out by committees composed of WHI investigators and NHLBI representatives. Open access publishing was funded by the German Research Foundation (DFG) and the University of Würzburg.
Competing interests None declared.

Patient consent for publication Not required.

Provenance and peer review Not commissioned; externally peer reviewed.

Data sharing statement The data, analytic methods and study materials are made available to other researchers for purposes of reproducing the results or replicating the procedure. The data underlying our work can be obtained from BioLINCC, a repository maintained by the National Heart, Lung, and Blood Institute. The BioLINCC website (https://biolincc.nhlbi.nih.gov/home/) includes detailed information about the available data and the process to obtain such data.

Open access This is an open access article distributed in accordance with the Creative Commons Attribution Non Commercial (CC BY-NC 4.0) license, which permits others to distribute, remix, adapt, build upon this work non-commercially, and license their derivative works on different terms, provided the original work is properly cited, appropriate credit is given, any changes made indicated, and the use is non-commercial. See: http://creativecommons.org/licenses/by-nc/4.0/.

\section{REFERENCES}

1. Frank $S$, Durovic $S$, Kostner GM. The assembly of lipoprotein $L p(a)$. Eur J Clin Invest 1996;26:109-14.

2. Kamstrup PR, Tybjaerg-Hansen A, Steffensen R, et al. Genetically elevated lipoprotein(a) and increased risk of myocardial infarction. JAMA 2009;301:2331-9.

3. Nordestgaard BG, Langsted A. Lipoprotein (a) as a cause of cardiovascular disease: insights from epidemiology, genetics, and biology. J Lipid Res 2016;57:1953-75.

4. Boffa MB, Koschinsky ML. Lipoprotein (a): truly a direct prothrombotic factor in cardiovascular disease? $J$ Lipid Res 2016;57:745-57.

5. Anglés-Cano E, de la Peña Díaz A, Loyau S. Inhibition of fibrinolysis by lipoprotein(a). Ann N Y Acad Sci 2001;936:261-75.

6. O'Keefe RJ. Fibrinolysis as a target to enhance fracture healing. $N$ Engl J Med 2015;373:1776-8.

7. Kamstrup PR, Tybjærg-Hansen A, Nordestgaard BG. Elevated lipoprotein(a) and risk of aortic valve stenosis in the general population. J Am Coll Cardiol 2014;63:470-7.

8. Kronenberg F, Kuen E, Ritz E, et al. Lipoprotein(a) serum concentrations and apolipoprotein(a) phenotypes in mild and moderate renal failure. J Am Soc Nephrol 2000;11:105-15.

9. Orozco P. Atherogenic lipid profile and elevated lipoprotein (a) are associated with lower bone mineral density in early postmenopausal overweight women. Eur J Epidemiol 2004;19:1105-12.

10. Pliatsika P, Antoniou A, Alexandrou A, et al. Serum lipid levels and bone mineral density in Greek postmenopausal women. Gynecol Endocrinol 2012;28:655-60.

11. Design of the Women's Health Initiative clinical trial and observational study. The Women's Health Initiative Study Group. Control Clin Trials 1998;19:61-109.

12. Vu Dac N, Mezdour H, Parra HJ, et al. A selective bi-site immunoenzymatic procedure for human Lp[a] lipoprotein quantification using monoclonal antibodies against apo[a] and apoB. $J$ Lipid Res 1989;30:1437-43.

13. Myers GL, Cooper GR, Winn CL, et al. The centers for disease control-national heart, lung and blood institute lipid standardization program. an approach to accurate and precise lipid measurements. Clin Lab Med 1989;9:105-36.

14. Chang PY, Gold EB, Cauley JA, et al. Triglyceride levels and fracture risk in midlife women: Study of Women's Health Across the Nation (SWAN). J Clin Endocrinol Metab 2016;101:3297-305.

15. Yamauchi M, Yamaguchi T, Nawata K, et al. Increased low-density lipoprotein cholesterol level is associated with non-vertebral fractures in postmenopausal women. Endocrine 2015;48:279-86.

16. Cui LH, Shin MH, Chung EK, et al. Association between bone mineral densities and serum lipid profiles of pre- and post-menopausal rural women in South Korea. Osteoporos Int 2005;16:1975-81.

17. Ghadiri-Anari A, Mortezaii-Shoroki Z, Modarresi M, et al. Association of lipid profile with bone mineral density in postmenopausal women in Yazd province. Int J Reprod Biomed 2016;14:597-602.

18. Makovey J, Chen JS, Hayward C, et al. Association between serum cholesterol and bone mineral density. Bone 2009;44:208-13.

19. Yamaguchi T, Sugimoto T, Yano S, et al. Plasma lipids and osteoporosis in postmenopausal women. Endocr J 2002;49:211-7.

20 Jeong IK, Cho SW, Kim SW, et al. Lipid profiles and bone mineral density in pre- and postmenopausal women in Korea. Calcif Tissue Int 2010;87:507-12.

21. Wang Z, Li Y, Zhou F, et al. Effects of statins on bone mineral density and fracture risk: a PRISMA-compliant systematic review and metaanalysis. Medicine 2016;95:e3042. 
22. Anagnostis $\mathrm{P}$, Galanis $\mathrm{P}$, Chatzistergiou $\mathrm{V}$, et al. The effect of hormone replacement therapy and tibolone on lipoprotein (a) concentrations in postmenopausal women: a systematic review and meta-analysis. Maturitas 2017;99:27-36.

23. Suk Danik J, Rifai N, Buring JE, et al. Lipoprotein(a), hormone replacement therapy, and risk of future cardiovascular events. J Am Coll Cardiol 2008;52:124-31.
24 Erqou S, Thompson A, Di Angelantonio E, et al. Apolipoprotein(a) isoforms and the risk of vascular disease: systematic review of 40 studies involving 58,000 participants. J Am Coll Cardiol 2010:55:2160-7.

25 Saleheen D, Haycock PC, Zhao W, et al. Apolipoprotein(a) isoform size, lipoprotein(a) concentration, and coronary artery disease: a mendelian randomisation analysis. Lancet Diabetes Endocrinol 2017;5:524-33. 